\title{
Invertibility in tensor products of Q-algebras
}

\author{
by \\ Seán Dineen (Dublin) and Pablo Sevilla-Peris (Valencia)
}

\begin{abstract}
We consider, using various tensor norms, the completed tensor product of two unital lmc algebras one of which is commutative. Our main result shows that when the tensor product of two Q-algebras is an lmc algebra, then it is a Q-algebra if and only if pointwise invertibility implies invertibility (as in the Gelfand theory). This is always the case for Fréchet algebras.
\end{abstract}

In 1973 L. Waelbroeck [26] introduced the concept of Banach-valued spectrum for commutative unital Banach algebras using the projective tensor product. This concept has been extended by various authors in the last twenty five years and more recently in a systematic fashion by S. Dineen, R. E. Harte and C. Taylor [9]-[11]. These authors considered arbitrary tensor norms and arbitrary unital Banach algebras. In this article we extend the scope of this investigation to wider collections of algebras. Initially we considered arbitrary locally multiplicatively convex (lmc) algebras, but as our investigations proceeded we found that the main results centered around Fréchet and Q-algebras. For these overlapping collections we prove \{pointwise invertibility $\} \Rightarrow$ invertibility $\}$ results using different methods. In the Fréchet algebra case we use techniques due to R. Arens [3], and for the Q-algebra case we develop and apply a vector-valued spectral theory following [9]-[11]. By combining the two cases we obtain necessary and sufficient conditions under which certain tensor products of Q-algebras are Q-algebras.

In $\S 1$ we define uniform tensor norms and topologies, lmc algebras and Q-algebras and give a number of examples. In $\S 2$ we define a number of vector-valued spectra and discuss their basic properties. We specialize to algebra-valued spectra in $\S 3$ and prove invertibility results. In $\S 4$ we use the Gelfand transform to obtain further criteria for invertibility.

2000 Mathematics Subject Classification: Primary 46H05.

Key words and phrases: Q-algebra, left inverse, uniform tensor norm.

The second author was supported by a grant of the Ministerio de Educación y Cultura (Spain), FP-97 29183763. 
We refer to A. Defant and K. Floret [7] and H. Jarchow [18] for tensor products and to A. Mallios [20] and A. E. Michael [22] for the theory of topological algebras. The authors wish to thank Domingo García and Manuel Maestre for fruitful conversations and Maria Fragoulopoulou for helpful comments and for drawing our attention to relevant literature.

\section{Uniform tensor norms and Q-algebras}

Definition 1.1. A method $\tau$ of assigning a norm \|\|$_{\tau}$ on $E \otimes F$ to every pair of normed linear spaces is called a uniform tensor norm if the following hold:

(1) for normed linear spaces $E, F,\|x \otimes y\|_{\tau}=\|x\| \cdot\|y\|$ for $x \in E$ and $y \in F$,

(2) for normed linear spaces $E_{i}, F_{i}, i=1,2$, and $T_{i} \in \mathcal{L}\left(E_{i} ; F_{i}\right)$ (continuous linear mappings from $E_{i}$ to $\left.F_{i}\right), i=1,2$, we have $T_{1} \otimes T_{2} \in$ $\mathcal{L}\left(E_{1} \otimes_{\tau} E_{2} ; F_{1} \otimes_{\tau} F_{2}\right)$ and

$$
\left\|T_{1} \otimes T_{2}\right\| \leq\left\|T_{1}\right\| \cdot\left\|T_{2}\right\| .
$$

A uniform tensor norm satisfies the projective limit condition if for any set of projective limits

$$
\varliminf_{i} E_{i}=\varliminf_{j} \lim _{j} F_{j}, \quad \varliminf_{\alpha}^{\lim } G_{\alpha}=\varliminf_{\beta}^{\lim _{\beta}} H_{\beta}
$$

of normed linear spaces we have

$$
\varliminf_{i, \alpha}\left(E_{i} \otimes_{\tau} G_{\alpha}\right)=\varliminf_{j, \beta}^{\lim _{j}}\left(F_{j} \otimes_{\tau} H_{\beta}\right)
$$

When the projective limit condition is satisfied we let

$$
\left(\varliminf_{i} E_{i}\right) \otimes_{\tau}\left(\varliminf_{\alpha}^{\lim } G_{\alpha}\right)=\varliminf_{i, \alpha}^{\lim }\left(E_{i} \otimes_{\tau} G_{\alpha}\right) \text {. }
$$

For locally convex spaces we let $E \widehat{\otimes}_{\tau} F$ denote the completion of $E \otimes_{\tau} F$. The projective $(\pi)$ and injective $(\varepsilon)$ norms are uniform tensor norms which satisfy the projective limit condition. Further examples are given by the Lapresté tensor norms [7, §12.5 and §12.7].

If $\tau$ is a uniform tensor norm which satisfies the projective limit condition then

(1) for any pair of locally convex spaces the canonical bilinear mapping $E \times F \rightarrow E \otimes_{\tau} F$ is separately continuous,

(2) if $E_{i}, F_{i}, i=1,2$, are locally convex spaces and $M_{i} \subseteq \mathcal{L}\left(E_{i} ; F_{i}\right)$, $i=1,2$, are equicontinuous sets, then $M_{1} \otimes M_{2} \subseteq \mathcal{L}\left(E_{1} \otimes_{\tau} E_{2} ; F_{1} \otimes_{\tau} F_{2}\right)$ is an equicontinuous set.

All algebras considered are over the field of complex numbers. An algebra $\mathcal{A}$ which is also a locally convex space is a locally multiplicatively convex 
(lmc) algebra if its topology is generated by a family $\left(p_{\alpha}\right)_{\alpha \in \Gamma}$ of continuous seminorms such that

$$
p_{\alpha}(x \cdot y) \leq p_{\alpha}(x) \cdot p_{\alpha}(y)
$$

for all $\alpha \in \Gamma$ and all $x, y \in \mathcal{A}$.

If $\mathcal{A}$ is a complete lmc algebra then there exists a collection $\left(\mathcal{A}_{\alpha}\right)_{\alpha}$ of Banach algebras and continuous homomorphisms $\pi_{\alpha}: \mathcal{A} \rightarrow \mathcal{A}_{\alpha}$ such that $\pi_{\alpha}(\mathcal{A})$ is dense in $\mathcal{A}_{\alpha}$ and $\mathcal{A}=\lim _{\alpha}\left(\mathcal{A}_{\alpha}, \pi_{\alpha}\right)$ (a projective limit representation such that $\pi_{\alpha}(\mathcal{A})$ is dense in $\mathcal{A}_{\alpha}$ for all $\alpha$ is called reduced).

An algebra with identity $1_{\mathcal{A}}$ is called a unital algebra. We shall also use $1_{\mathcal{A}}$ to denote the identity operator on $\mathcal{A}$, i.e. $1_{\mathcal{A}}(x)=x$ for all $x \in \mathcal{A}$. A complete unital lmc algebra is a $Q$-algebra if the set $\mathcal{A}_{\text {inv }}$ of invertible elements in $\mathcal{A}$ is open (a more general definition of Q-algebra is given in [20] but we have used the above for convenience). This is the case if and only if the identity has a neighbourhood consisting of invertible elements. The spectrum of $\mathcal{A}, \mathfrak{M}(\mathcal{A})$ (the set of all continuous non-zero $\mathbb{C}$-valued homomorphisms on $\mathcal{A}$ ), is a weak*-compact subset of $\mathcal{A}^{\prime}$ when $\mathcal{A}$ is a $\mathrm{Q}$ algebra ([27, Proposition 10]). All unital Banach algebras are Q-algebras. An important classical example of a Q-algebra which is not a Banach algebra is the space $\mathcal{C}^{\infty}[0,1]$ endowed with the topology of uniform convergence of functions and all their derivatives over $[0,1]$.

We now connect the concepts of tensor product and lmc algebra. If $\mathcal{A}$ and $\mathcal{B}$ are algebras then universal properties of the tensor product show that the product

$$
\left(a_{1} \otimes b_{1}\right) \cdot\left(a_{2} \otimes b_{2}\right):=a_{1} a_{2} \otimes b_{1} b_{2}
$$

for $a_{i} \in \mathcal{A}$ and $b_{i} \in \mathcal{B}$ extends to define a product on the algebra $\mathcal{A} \otimes \mathcal{B}$ $([9,20])$.

Definition 1.2. If $\mathcal{A}$ and $\mathcal{B}$ are complete lmc algebras and $\tau$ is a uniform tensor norm which satisfies the projective limit condition, then we say that $\mathcal{A} \widehat{\otimes}_{\tau} \mathcal{B}$ is representable if there exist reduced projective Banach algebra representations $\mathcal{A}=\lim _{\alpha}\left(\mathcal{A}_{\alpha}, \pi_{\alpha}\right)$ and $\mathcal{B}=\lim _{\beta}\left(\mathcal{B}_{\beta}, \varrho_{\beta}\right)$ such that $\mathcal{A}_{\alpha} \widehat{\otimes}_{\tau} \mathcal{B}_{\beta}$ is a Banach algebra for all $\alpha$ and $\beta$.

If $\mathcal{A} \widehat{\otimes}_{\tau} \mathcal{B}$ is representable then, by the projective limit condition, $\mathcal{A} \widehat{\otimes}_{\tau} \mathcal{B}=\lim _{\alpha, \beta}\left(\mathcal{A}_{\alpha} \widehat{\otimes}_{\tau} \mathcal{B}_{\beta}, \pi_{\alpha} \otimes \varrho_{\beta}\right)$ is a complete lmc algebra.

Results in [9] show that $\mathcal{A} \widehat{\otimes}_{\pi} \mathcal{B}$ is representable for any pair of lmc algebras $\mathcal{A}$ and $\mathcal{B}$, and $\mathcal{A} \widehat{\otimes}_{\varepsilon} \mathcal{B}$ is representable if there exists a reduced projective limit representation $\mathcal{A}=\lim _{\alpha}\left(\mathcal{A}_{\alpha}, \pi_{\alpha}\right)$ where each $\mathcal{A}_{\alpha}$ is a uniform Banach algebra and $\mathcal{B}$ is an lmc algebra. Further examples can be obtained using Lapresté's tensor norms [7, §12.5 and §12.7].

We call a complete lmc algebra whose topology is generated by a countable set of seminorms a Fréchet algebra; a more general definition is given 
in [20]. Thus every Fréchet algebra has a countable reduced projective representation by Banach algebras and continuous homomorphisms. An element $a$ in a unital algebra is left invertible if there exists $b \in \mathcal{A}$ such that $b \cdot a=1_{\mathcal{A}}$. The following result for unital lmc algebras is due to M. Fragoulopoulou ([13, Lemma 6.8] and [14, Proposition 4. 13]). For the sake of completeness we include a proof for Fréchet algebras, a case that we require later.

Proposition 1.3. If $\mathcal{A}$ and $\mathcal{B}$ are unital Fréchet algebras with $\mathcal{A}$ commutative, $\tau$ is a uniform tensor norm that satisfies the projective limit condition and $\mathcal{A} \widehat{\otimes}_{\tau} \mathcal{B}$ is representable then the following are equivalent for $a \in \mathcal{A} \widehat{\otimes}_{\tau} \mathcal{B}:$

(1) a is left invertible in $\mathcal{A} \widehat{\otimes}_{\tau} \mathcal{B}$.

$(2)\left(h \otimes I_{\mathcal{B}}\right)($ a) is left invertible for all $h \in \mathfrak{M}(\mathcal{A})$.

Proof. Since algebra homomorphisms map left invertibles to left invertibles we clearly have $(1) \Rightarrow(2)$.

Now suppose that (2) is satisfied. By our hypothesis we have $\mathcal{A}=$ $\lim _{n}\left(\mathcal{A}_{n}, \pi_{n}\right), \mathcal{B}=\lim _{n}\left(\mathcal{B}_{n}, \varrho_{n}\right)$ and $\mathcal{A} \widehat{\otimes}_{\tau} \mathcal{B}=\lim _{n}\left(\mathcal{A}_{n} \widehat{\otimes}_{\tau} \mathcal{B}_{n}, \pi_{n} \otimes \varrho_{n}\right)$ where each $\mathcal{A}_{n} \widehat{\otimes}_{\tau} \overleftarrow{\mathcal{B}_{n}}$ is a Banach algebra and $\pi_{n} \overleftarrow{\otimes} \varrho_{n}\left(\mathcal{A} \widehat{\otimes}_{\tau} \mathcal{B}\right)$ is dense in $\mathcal{A}_{n} \widehat{\otimes}_{\tau} \mathcal{B}_{n}$.

If $h_{n} \in \mathfrak{M}\left(\mathcal{A}_{n}\right)$ then $h_{n} \circ \pi_{n} \in \mathfrak{M}(\mathcal{A})$ and, by $(2),\left(\left(h_{n} \circ \pi_{n}\right) \otimes I_{\mathcal{B}}\right)($ a) is left invertible in $\mathcal{B}$. Hence $\varrho_{n}\left(\left(h_{n} \circ \pi_{n}\right) \otimes I_{\mathcal{B}}(\right.$ a $\left.)\right)$ is left invertible in $\mathcal{B}_{n}$. By first considering elements of $\mathcal{A} \otimes \mathcal{B}$ and then using continuity we see that

$$
\varrho_{n}\left(\left(h_{n} \circ \pi_{n}\right) \otimes I_{\mathcal{B}}(\mathrm{a})\right)=\left(h_{n} \otimes I_{\mathcal{B}}\right)\left(\pi_{n} \otimes \varrho_{n}(\mathrm{a})\right) .
$$

By [9, Proposition 20], $\pi_{n} \otimes \varrho_{n}\left(\right.$ a) is left invertible in $\mathcal{A}_{n} \widehat{\otimes}_{\tau} \mathcal{B}_{n}$, and by [3, Theorem 4.2], a is left invertible in $\mathcal{A} \widehat{\otimes}_{\tau} \mathcal{B}$.

EXAMPLE 1.4. Let $X$ be a completely regular hemicompact $k_{\mathbb{R}}$-space (see [16, Chapter 3], [20, Theorem 1.2, p. 223]). Then $\mathcal{C}(X)$, the complexvalued functions on $X$ endowed with the compact open topology, is a unital Fréchet algebra and $\mathfrak{M}(\mathcal{C}(X))$ can be identified with point evaluations at points of $X([16,4.1 .7])$. If $\mathcal{B}$ is unital Fréchet algebra then $\mathcal{C}(X, \mathcal{B}) \cong$ $\mathcal{C}(X) \widehat{\otimes}_{\varepsilon} \mathcal{B}([18,16.6 .3])$. If $\delta_{x}$ is point evaluation at $x \in X$ then, by the above identification $\delta_{x} \otimes I_{\mathcal{B}}(f)=f(x)$ for all $f \in \mathcal{C}(X, \mathcal{B})$. By Proposition 1.3, $f \in \mathcal{C}(X, \mathcal{B})$ is left invertible if and only if $f(x)$ is left invertible in $\mathcal{B}$ for all $x \in X$. This result is a special case of [8, Proposition 1] due to S. Dierolf and K. Aye Aye.

We remark that the left inverse of an element of an algebra is not necessarily unique and thus this result says that we may make a continuous selection of left inverses. The same result is true for right inverses and combining these two cases we obtain the result for inverses. However, the result for inverses is trivial since $f^{-1}(x):=(f(x))^{-1}$ defines a continuous inverse. 
EXAMPLE 1.5. Let $U$ denote a connected pseudo-convex Riemann domain over $E \cong F_{\mathrm{c}}^{\prime}$, where $F_{\mathrm{c}}^{\prime}$ is endowed with the compact open topology and $F$ is a Fréchet space with the approximation property. Results by J. Mujica [23] and M. Schottenloher [24] show that $\left(H(U), \tau_{0}\right)$ is a Fréchet space and $\mathfrak{M}\left(H(U), \tau_{0}\right)=U$ (via point evaluations). Note that if $F$ is separable then $U$ is a hemicompact topological space but, in general, $U$ will not be hemicompact. We have

$$
\left(H(U, \mathcal{B}), \tau_{0}\right) \cong\left(H(U), \tau_{0}\right) \widehat{\otimes}_{\varepsilon} \mathcal{B}
$$

for any unital Banach algebra $\mathcal{B}([12])$. Using the method of the previous example and Proposition 1.3 we see that if $f \in H(U, \mathcal{B})$ is left invertible at each point, i.e. if $f(z) \in \mathcal{B}$ is left invertible for all $z \in U$, then there exists $g \in H(U, \mathcal{B})$ such that $g(z) f(z)=1_{\mathcal{B}}$ (identity on $\mathcal{B}$ ) for all $z \in U$. This result is due to G. Allan [1], [2] when $E$ is finite-dimensional. An example in [6] shows that the pseudo-convexity condition is necessary. Connectedness can be removed by considering each connected component separately.

2. Vector-valued spectra. We require three different left spectra in this article. For the reader's convenience we collect here the notation that we shall subsequently use. If $\mathcal{A}$ is a unital lmc algebra, $E$ is a locally convex space and $\tau$ is a uniform tensor norm we let:

- $\sigma_{\mathrm{H}}^{\text {left }}(\mathrm{a})$ denote the left joint Harte spectrum of a collection a $:=\left(a_{i}\right)_{i \in I}$

- $\sigma^{\text {left }}(a)$ denote the vector-valued left spectrum of $a \in \mathcal{A} \widehat{\otimes}_{\tau} E$, and

- $\sigma_{\mathcal{A}}^{\text {left }}(a)$ denote the (usual) left spectrum of a $\in \mathcal{A}$.

In this section we develop a theory of vector-valued spectra similar to that developed for Banach algebras in [9]-[11]. We use this theory to extend Proposition 1.3 to Q-algebras in $\S 3$.

Definition 2.1. For a family a $=\left(a_{i}\right)_{i \in I} \subseteq \mathcal{A}$, a unital lme algebra, the left joint Harte spectrum $\sigma_{\mathrm{H}}^{\text {left }}(\mathrm{a})$ of a is the set of all $\left(\lambda_{i}\right)_{i \in I} \in \mathbb{C}^{I}$ such that

$$
1_{\mathcal{A}} \notin\left\{\sum_{\substack{i \in F \subseteq I \\ F \text { finite }}} b_{i}\left(a_{i}-\lambda_{i} 1_{\mathcal{A}}\right): b_{i} \in \mathcal{A}\right\} .
$$

Note that the set of all finite sums above is the left ideal generated by $\left(a_{i}\right)_{i \in I}$. When the family consists of just one element, the joint left spectrum is the scalar left spectrum. If $\lambda:=\left(\lambda_{i}\right)_{i \in I} \in \mathbb{C}^{I}$ then $\lambda \in \sigma_{\mathrm{H}}^{\text {left }}(\mathrm{a})$ if and only if $\left(\lambda_{j}\right)_{j \in J} \in \sigma_{\mathrm{H}}^{\text {left }}\left(\left(a_{j}\right)_{j \in J}\right)$ for each finite subset $J$ of $I$. If the indexing set $I$ is a locally convex space $E$, then we can interpret a $:=\left(a_{x}\right)_{x \in E}$ as a mapping a $: E \rightarrow \mathcal{A}$ by letting a $(x)=a_{x}$, and $\lambda=\left(\lambda_{x}\right)_{x \in E}$ as a mapping $\lambda: E \rightarrow \mathbb{C}$ by letting $\lambda(x)=\lambda_{x}$. Under certain conditions, properties of a as a mapping are inherited by elements of the spectrum. 
Lemma 2.2. If $\mathcal{A}$ is a Q-algebra, a $\in \mathcal{L}(E ; \mathcal{A})$ and $\lambda \in \sigma_{\mathrm{H}}^{\text {left }}(\mathrm{a})$, then $\lambda \in E^{\prime}$. If $E=\mathcal{B}$ is an lmc algebra and a is a non-zero algebra homomorphism, then so also is $\lambda \in \sigma_{\mathrm{H}}^{\text {left }}(\mathrm{a})$.

Proof. The algebraic properties follow from the proof of [9, Lemma 3]. It remains to show that $\lambda$ is continuous. Let $\left(x_{\alpha}\right)_{\alpha} \subseteq E$ and suppose $x_{\alpha} \rightarrow x$ as $\alpha \rightarrow \infty$. If $\lambda \in \sigma_{\mathrm{H}}^{\text {left }}(\mathrm{a})$ and $\lambda\left(x_{\alpha}\right) \nrightarrow \lambda(x)$ as $\alpha \rightarrow \infty$ then we can find $\delta>0$ such that (on taking a subnet if necessary) $\left|\lambda\left(x_{\alpha}\right)-\lambda(x)\right| \geq \delta$ for all $\alpha$. We have

$$
\left(\mathrm{a}\left(x_{\alpha}\right)-\lambda\left(x_{\alpha}\right) 1_{\mathcal{A}}\right)-\left(\mathrm{a}(x)-\lambda(x) 1_{\mathcal{A}}\right)=\left(\lambda(x)-\lambda\left(x_{\alpha}\right)\right)\left(\frac{\mathrm{a}\left(x_{\alpha}\right)-\mathrm{a}(x)}{\lambda(x)-\lambda\left(x_{\alpha}\right)}+1_{\mathcal{A}}\right)
$$

for all $\alpha$. Since $\mathcal{A}$ is a Q-algebra there exists a neighbourhood $U$ of 0 such that $1_{\mathcal{A}}+U \subseteq \mathcal{A}_{\text {inv }}$. For $\alpha$ sufficiently large

$$
\frac{a_{x_{\alpha}}-a_{x}}{\lambda(x)-\lambda\left(x_{\alpha}\right)} \in U
$$

and hence $\left(\mathrm{a}\left(x_{\alpha}\right)-\lambda\left(x_{\alpha}\right) 1_{\mathcal{A}}\right)-\left(\mathrm{a}(x)-\lambda(x) 1_{\mathcal{A}}\right)$ is invertible. This contradicts the fact that $\lambda \in \sigma_{\mathrm{H}}^{\text {left }}(\mathrm{a})$. Hence $\lambda$ is continuous.

If $a$ is an algebra homomorphism then so also is $\lambda$ by [9, Lemma 3]. If $\mathrm{a} \neq 0$ then $\mathrm{a}\left(1_{\mathcal{B}}\right)=1_{\mathcal{A}}$ and

$$
1_{\mathcal{A}} \in\left\{\sum_{\substack{i \in F \subseteq \mathcal{B} \\ F \text { finite }}} b_{i} \mathrm{a}(i): b_{i} \in \mathcal{A}\right\}
$$

and $\lambda \equiv 0$ does not belong to $\sigma_{\mathrm{H}}^{\text {left }}(\mathrm{a})$. This contradicts the fact that $\lambda \in$ $\sigma_{\mathrm{H}}^{\text {left }}(\mathrm{a})$ and completes the proof.

Definition 2.3. Let $\mathcal{A}$ be a unital lmc algebra, $E$ a locally convex space and let $\tau$ be a uniform tensor norm on $\mathcal{A} \otimes E$. If a $\in \mathcal{A} \widehat{\otimes}_{\tau} E$ we define the left vector spectrum $\sigma^{\text {left }}(\mathrm{a})$ of a to be $\sigma_{\mathrm{H}}^{\text {left }}\left(\left\{\left[1_{\mathcal{A}} \otimes x^{\prime}\right](\mathrm{a})\right\}_{x^{\prime} \in E^{\prime}}\right)$.

By Lemma 2.2,

$$
\begin{aligned}
\sigma^{\text {left }}(\mathrm{a})=\left\{x^{\prime \prime} \in E^{\prime \prime}\right. & : \\
1_{\mathcal{A}} & \left.\neq \sum_{\substack{i \in F \\
F \text { finite }}} b_{i}\left(\left[1_{\mathcal{A}} \otimes x_{i}^{\prime}\right](\mathrm{a})-x^{\prime \prime}\left(x_{i}^{\prime}\right) 1_{\mathcal{A}}\right), b_{i} \in \mathcal{A}, x_{i}^{\prime} \in E^{\prime}\right\} .
\end{aligned}
$$

We now prove results which allow us to rewrite $\sigma^{\text {left }}(\mathrm{a})$ in a more convenient fashion. Let $J_{E}$ denote the canonical mapping from a locally convex space into its bidual. The following result extends [9, Proposition 6] from unital Banach algebras to Q-algebras.

Proposition 2.4. Let $\mathcal{A}$ be a Q-algebra, $E$ a complete locally convex space and $\tau$ a uniform tensor topology. If $\mathrm{a} \in \mathcal{A} \widehat{\otimes}_{\tau} E$ and $x^{\prime \prime} \in \sigma^{\text {left }}(\mathrm{a})$ then $x^{\prime \prime}=J_{E}(x)$ for some $x \in E$. 
Proof. If $\mathrm{a} \in \mathcal{A} \otimes E$ then the mapping

$$
x^{\prime} \in\left(E^{\prime}, \sigma\left(E^{\prime}, E\right)\right) \mapsto\left[1_{\mathcal{A}} \otimes x^{\prime}\right](\mathrm{a}) \in \mathcal{A}
$$

is easily seen to be continuous.

Let $M$ denote a closed equicontinuous subset of $E^{\prime}$ and suppose $\left(x_{\alpha}^{\prime}\right)_{\alpha} \subseteq$ $M$ converges in the $\sigma\left(E^{\prime}, E\right)$ topology to $x^{\prime} \in M$ as $\alpha \rightarrow \infty$. Let $p$ denote a continuous seminorm on $\mathcal{A}$ and let $\varepsilon>0$ be arbitrary. Since $\left\{1_{\mathcal{A}}\right\} \otimes M \subseteq$ $\mathcal{L}\left(\mathcal{A} \widehat{\otimes}_{\tau} E ; \mathcal{A}\right)$ is equicontinuous there exists a continuous seminorm $q$ on $\mathcal{A} \widehat{\otimes}_{\tau} E$ such that

$$
\sup _{x^{\prime} \in M} p\left(\left[1_{\mathcal{A}} \otimes x^{\prime}\right](\mathrm{b})\right) \leq q(\mathrm{~b})
$$

for all $\mathrm{b} \in \mathcal{A} \widehat{\otimes}_{\tau} E$. Now choose $\mathrm{b} \in \mathcal{A} \otimes E$ such that $q(\mathrm{a}-\mathrm{b})<\varepsilon$. Since $\mathrm{b}$ is a finite tensor there exists $\alpha_{0}$ such that

$$
p\left(\left[1_{\mathcal{A}} \otimes x_{\alpha}^{\prime}\right](\mathrm{b})-\left[1_{\mathcal{A}} \otimes x^{\prime}\right](\mathrm{b})\right)<\varepsilon
$$

for all $\alpha \geq \alpha_{0}$. By (2) and (3),

$p\left(\left[1_{\mathcal{A}} \otimes x_{\alpha}^{\prime}\right](\mathrm{a})-\left[1_{\mathcal{A}} \otimes x^{\prime}\right](\mathrm{a})\right) \leq p\left(\left[1_{\mathcal{A}} \otimes x_{\alpha}^{\prime}\right](\mathrm{b})-\left[1_{\mathcal{A}} \otimes x^{\prime}\right](\mathrm{b})\right)+2 q(\mathrm{a}-\mathrm{b}) \leq 3 \varepsilon$ for all $\alpha \geq \alpha_{0}$. Hence the mapping $x^{\prime} \in E^{\prime} \mapsto\left[1_{\mathcal{A}} \otimes x^{\prime}\right](\mathrm{a})$ is $\sigma\left(E^{\prime}, E\right)$ continuous on equicontinuous subsets of $E^{\prime}$.

Let $x^{\prime \prime} \in \sigma^{\text {left }}\left(\right.$ a). Suppose $\left(x_{\alpha}^{\prime}\right)_{\alpha}$ is an equicontinuous net in $E^{\prime}$ which converges to $x^{\prime} \in E^{\prime}$ in the $\sigma\left(E^{\prime}, E\right)$ topology as $\alpha \rightarrow \infty$. If $x^{\prime \prime}\left(x_{\alpha}^{\prime}\right)$ does not converge to $x^{\prime \prime}\left(x^{\prime}\right)$, then, by taking a subnet if necessary, we can suppose there exists $\delta>0$ such that $\left|x^{\prime \prime}\left(x_{\alpha}^{\prime}\right)-x^{\prime \prime}\left(x^{\prime}\right)\right|>\delta$ for all $\alpha$. Since $\mathcal{A}$ is a Q-algebra we can choose a convex balanced neighbourhood $U$ of 0 such that $1_{\mathcal{A}}+U \subseteq \mathcal{A}_{\text {inv }}$. Since $x^{\prime} \mapsto\left[1_{\mathcal{A}} \otimes x^{\prime}\right](\mathrm{a})$ is $\sigma\left(E^{\prime}, E\right)$-continuous on equicontinuous sets, there exists $\alpha_{0}$ such that

$$
-\left[1_{\mathcal{A}} \otimes\left(x_{\alpha}^{\prime}-x^{\prime}\right)\right](\mathrm{a}) \in \delta U
$$

for all $\alpha \geq \alpha_{0}$. Hence

$$
\begin{aligned}
{\left[1_{\mathcal{A}} \otimes\left(x_{\alpha}^{\prime}-x^{\prime}\right)\right](\mathrm{a}) } & -x^{\prime \prime}\left(x_{\alpha}^{\prime}-x^{\prime}\right) 1_{\mathcal{A}} \\
= & -x^{\prime \prime}\left(x_{\alpha}^{\prime}-x^{\prime}\right)\left(1_{\mathcal{A}}-\frac{\left[1_{\mathcal{A}} \otimes\left(x_{\alpha}^{\prime}-x^{\prime}\right)\right](\mathrm{a})}{x^{\prime \prime}\left(x_{\alpha}^{\prime}-x^{\prime}\right)}\right) \in \mathcal{A}_{\mathrm{inv}} .
\end{aligned}
$$

This contradicts the fact that $x^{\prime \prime} \in \sigma^{\text {left }}(\mathrm{a})$. Hence $x^{\prime \prime}$ is $\sigma\left(E^{\prime}, E\right)$-continuous on equicontinuous sets of $E^{\prime}$, and Grothendieck's completeness criterion [17, Chapter 4, Section 11, Corollary 3] implies $x^{\prime \prime} \in J_{E}(x)$ for some $x \in E$.

Because of Proposition 2.4 we identify $\sigma^{\text {left }}($ a) with a subset of $E$ for any $a \in \mathcal{A} \widehat{\otimes}_{\tau} E$.

By first considering elements of $\mathcal{A} \otimes E$ and then using density and continuity we see that 


$$
\begin{aligned}
b\left(\left[1_{\mathcal{A}} \otimes x^{\prime}\right](\mathrm{a})-x^{\prime}(x) 1_{\mathcal{A}}\right) & =b\left(\left[1_{\mathcal{A}} \otimes x^{\prime}\right](\mathrm{a})-\left[1_{\mathcal{A}} \otimes x^{\prime}\right]\left(1_{\mathcal{A}} \otimes x\right)\right) \\
& =b\left(\left[1_{\mathcal{A}} \otimes x^{\prime}\right]\left(\mathrm{a}-1_{\mathcal{A}} \otimes x\right)\right) \\
& =\left[b \otimes x^{\prime}\right]\left(\mathrm{a}-1_{\mathcal{A}} \otimes x\right)
\end{aligned}
$$

for $b \in \mathcal{A}, x^{\prime} \in E^{\prime}, x \in E$ and $\mathrm{a} \in \mathcal{A} \widehat{\otimes}_{\tau} E$. This allows us to rewrite $\sigma^{\text {left }}(\mathrm{a})$, a $\in \mathcal{A} \widehat{\otimes}_{\tau} E$ for $\mathcal{A}$ a Q-algebra, $E$ a complete locally convex space and $\tau$ a uniform tensor norm, as follows:

$$
\begin{aligned}
& \sigma^{\text {left }}(\mathrm{a}) \\
& =\left\{x \in E: 1_{\mathcal{A}} \notin\left\{\sum_{\substack{i \in F \\
F \text { finite }}} a_{i}\left(\left[1_{\mathcal{A}} \otimes x_{i}^{\prime}\right]\left(\mathrm{a}-1_{\mathcal{A}} \otimes x\right)\right): a_{i} \in \mathcal{A}, x_{i} \in E^{\prime}\right\}\right\} \\
& =\left\{x \in E: 1_{\mathcal{A}} \neq \mathrm{b} \cdot\left(\mathrm{a}-1_{\mathcal{A}} \otimes x\right) \text { for any } \mathrm{b} \in \mathcal{A} \otimes E^{\prime}\right\} .
\end{aligned}
$$

In (4), $\mathcal{A} \otimes E^{\prime}$ acts on $\mathcal{A} \otimes E$ by a linear extension of the action $\left(a \otimes x^{\prime}\right)$. $(b \otimes x):=x^{\prime}(x) a b$. This final description is similar in form to the classical one.

In the commutative case we recover the definition of Waelbroeck [26]. This extends the Banach algebra result in [9, Proposition 7].

Proposition 2.5. Let $\mathcal{A}$ be a commutative Q-algebra, $E$ a complete locally convex space and $\tau$ a uniform tensor norm. If $\mathrm{a} \in \mathcal{A} \widehat{\otimes}_{\tau} E$, then

$$
\sigma^{\text {left }}(\mathrm{a})=\left\{\left[h \otimes I_{E}\right](\mathrm{a}): h \in \mathfrak{M}(\mathcal{A})\right\} .
$$

Proof. Suppose $x \notin \sigma^{\text {left }}(\mathrm{a})$. Then there exists $\mathrm{b} \in \mathcal{A} \otimes E^{\prime}$ such that $\mathrm{b} \cdot\left(\mathrm{a}-1_{\mathcal{A}} \otimes x\right)=1_{\mathcal{A}}$. If $h \in \mathfrak{M}(\mathcal{A})$ then by first considering elements in $\mathcal{A} \otimes E$ and a density argument we see that

$$
h\left(\mathrm{~b} \cdot \mathrm{a}-1_{\mathcal{A}} \otimes x\right)=1=\left(h \otimes I_{E^{\prime}}(\mathrm{b})\right)\left(h \otimes I_{E}(\mathrm{a})-x\right) .
$$

Hence $x \neq h \otimes I_{E}(\mathrm{a})$ for any $h \in \mathfrak{M}(\mathcal{A})$ and $h \otimes I_{E}(\mathrm{a}) \in \sigma^{\text {left }}(\mathrm{a})$.

Conversely, if $x \in \sigma^{\text {left }}(\mathrm{a})$ then, as $\mathcal{A}$ is a Q-algebra and so all its maximal ideals are closed $\left(\left[22\right.\right.$, p. 80]), there is $h \in \mathfrak{M}(\mathcal{A})$ such that $h\left(\mathrm{~b} \cdot\left(\mathrm{a}-1_{\mathcal{A}} \otimes x\right)\right)$ $=0$ for all $\mathrm{b} \in \mathcal{A} \otimes E^{\prime}$. In particular taking $\mathrm{b}=1_{\mathcal{A}} \otimes x^{\prime}$ we obtain

$$
0=h\left(1_{\mathcal{A}} \otimes x^{\prime} \cdot \mathrm{a}-1_{\mathcal{A}} \otimes x\right)=x^{\prime}\left(h \otimes I_{E}(\mathrm{a})-x\right) .
$$

Since $x^{\prime}$ was arbitrary the Hahn-Banach theorem implies $\left[h \otimes I_{E}\right](\mathrm{a})=x$ and this completes the proof.

The following result generalizes [5, Proposition 2.3] to the non-commutative setting.

Proposition 2.6. Let $\mathcal{A}=\lim _{i}\left(\mathcal{A}_{i}, \pi_{i}\right)$ be a reduced projective limit representation of the $Q$-algebra $\mathcal{A}$ by complete unital lmc algebras $\mathcal{A}_{i}$ where each $\pi_{i}$ is an algebra homomorphism, let $E$ be a locally convex space and suppose $\tau$ is a uniform tensor norm which satisfies the projective limit condition. If 
$\mathrm{a} \in \mathcal{A} \widehat{\otimes}_{\tau} E$ and $\mathrm{a}_{i}=\pi_{i} \otimes I_{E}(\mathrm{a})$ for all $i \in I$ then

$$
\sigma^{\mathrm{left}}(\mathrm{a})=\bigcup_{i \in I} \sigma^{\mathrm{left}}\left(\mathrm{a}_{i}\right) \text {. }
$$

Proof. If $x \notin \sigma^{\text {left }}(\mathrm{a})$ then there is $\mathrm{b} \in \mathcal{A} \otimes E^{\prime}$ such that $\mathrm{b} \cdot\left(\mathrm{a}-1_{\mathcal{A}} \otimes x\right)$ $=1_{\mathcal{A}}$. By first considering elements of $\mathcal{A} \otimes E$ and then using density and continuity and the fact that the projective limit is reduced we obtain

$$
\begin{aligned}
1_{\mathcal{A}_{i}} & =\pi_{i}\left(1_{\mathcal{A}}\right)=\pi_{i}\left(\mathrm{~b} \cdot\left(\mathrm{a}-1_{\mathcal{A}} \otimes x\right)\right) \\
& =\left(\pi_{i} \otimes I_{E^{\prime}}\right)(\mathrm{b}) \cdot\left(\pi_{i} \otimes I_{E}(\mathrm{a})-\pi_{i}\left(1_{\mathcal{A}}\right) \otimes x\right) \\
& =\left(\pi_{i} \otimes I_{E^{\prime}}\right)(\mathrm{b}) \cdot\left(\mathrm{a}_{i}-1_{\mathcal{A}_{i}} \otimes x\right) .
\end{aligned}
$$

Since $\left(\pi_{i} \otimes I_{E^{\prime}}\right)(\mathrm{b}) \in \mathcal{A}_{i} \otimes E^{\prime}$ this implies $x \notin \sigma^{\text {left }}\left(\mathrm{a}_{i}\right)$ and

$$
\bigcup_{i \in I} \sigma^{\mathrm{left}}\left(\mathrm{a}_{i}\right) \subseteq \sigma^{\mathrm{left}}(\mathrm{a}) .
$$

Now suppose $x \in \sigma^{\text {left }}(\mathrm{a})$. Let $\mathcal{I}$ denote the left ideal in $\mathcal{A}$ generated by $\left\{\left(1_{\mathcal{A}} \otimes x^{\prime}\right) \cdot\left(\mathrm{a}-1_{\mathcal{A}} \otimes x\right)\right\}_{x^{\prime} \in E^{\prime}}$. Since $\mathcal{A}$ is a Q-algebra and $x \in \sigma^{\text {left }}(\mathrm{a})$ we have $1_{\mathcal{A}} \notin \overline{\mathcal{I}}$. By the Hahn-Banach theorem we can choose $\psi \in \mathcal{A}^{\prime}$ such that $\psi\left(1_{\mathcal{A}}\right)=1$ and $\psi(\overline{\mathcal{I}})=0$. By the projective limit representation there exist $i \in I$ and $\psi_{i} \in \mathcal{A}_{i}^{\prime}$ such that $\psi=\psi_{i} \circ \pi_{i}$. Since $\pi_{i}$ is an algebra homomorphism,

$$
\psi_{i}\left(1_{\mathcal{A}_{i}}\right)=\psi_{i}\left(\pi_{i}\left(1_{\mathcal{A}}\right)\right)=\psi\left(1_{\mathcal{A}}\right)=1
$$

Let $\mathcal{I}_{i}$ denote the left ideal in $\mathcal{A}_{i}$ generated by

$$
\left\{\left(1_{\mathcal{A}_{i}} \otimes x^{\prime}\right) \cdot\left(\mathrm{a}_{i}-1_{\mathcal{A}_{i}} \otimes x\right)\right\}_{x^{\prime} \in E^{\prime}}
$$

Since $\pi_{i}\left(\left(1_{\mathcal{A}} \otimes x^{\prime}\right) \cdot\left(\mathrm{a}-1_{\mathcal{A}} \otimes x\right)\right)=\left(1_{\mathcal{A}_{i}} \otimes x^{\prime}\right) \cdot\left(\mathrm{a}_{i}-1_{\mathcal{A}_{i}} \otimes x\right)$ we have $\pi_{i}(\mathcal{I}) \subseteq \mathcal{I}_{i}$ and $\overline{\pi_{i}(\mathcal{I})} \subseteq \overline{\mathcal{I}}_{i}$. Since the projective limit representation of $\mathcal{A}$ is reduced $\pi_{i}(\mathcal{A})$ is dense in $\mathcal{A}_{i}$. Let $b_{i} \in \mathcal{A}_{i}$ and suppose $\left(b_{\alpha}\right)_{\alpha} \subseteq \mathcal{A}$ and $\pi_{i}\left(b_{\alpha}\right) \rightarrow b_{i}$ as $\alpha \rightarrow \infty$. If $x^{\prime} \in E^{\prime}$ then

$$
\begin{aligned}
\left(b_{i} \otimes x^{\prime}\right) \cdot\left(\mathrm{a}_{i}-1_{\mathcal{A}_{i}} \otimes x\right) & =\lim _{\alpha}\left(\pi_{i}\left(b_{\alpha}\right) \otimes x^{\prime}\right) \cdot\left(\mathrm{a}_{i}-1_{\mathcal{A}_{i}} \otimes x\right) \\
& =\lim _{\alpha} \pi_{i}\left(b_{\alpha} \otimes x^{\prime}\right) \cdot\left(\mathrm{a}_{i}-1_{\mathcal{A}_{i}} \otimes x\right)
\end{aligned}
$$

and $\mathcal{I}_{i} \subseteq \overline{\pi_{i}(\mathcal{I})}$. Hence $\overline{\mathcal{I}}_{i}=\overline{\pi_{i}(\mathcal{I})}$.

If $w \in \overline{\mathcal{I}}_{i}$ then there exists $\left(w_{\beta}\right)_{\beta} \subseteq \mathcal{I}$ such that $w=\lim _{\beta} \pi_{i}\left(w_{\beta}\right)$. Hence

$$
\psi_{i}(w)=\psi_{i}\left(\lim _{\beta} \pi_{i}\left(w_{\beta}\right)\right)=\lim _{\beta} \psi_{i} \circ \pi_{i}\left(w_{\beta}\right)=\lim _{\beta} \psi\left(w_{\beta}\right)=0 .
$$

On the other hand $\psi_{i}\left(1_{\mathcal{A}_{i}}\right)=1$. Hence $\overline{\mathcal{I}}_{i}$ is a proper closed ideal in $\mathcal{A}_{i}$. This implies $x \in \sigma^{\text {left }}\left(\mathrm{a}_{i}\right)$ and completes the proof.

Our next example generalizes [3, Theorem 4.2] concerning invertibility in a projective limit of algebras. 
ExAmple 2.7. If $\mathcal{A}=\varliminf_{i} \mathcal{L}_{i}$ is a Q-algebra then $\mathcal{A} \widehat{\otimes}_{\tau} \mathbb{C} \cong \mathcal{A}$. Therefore, $\lambda \notin \sigma^{\text {left }}\left(a \otimes 1_{\mathbb{C}}\right)$ if and only if there is a finite sum, with $a_{i} \in \mathcal{A}$ and $\mu_{i} \in \mathbb{C}$, such that

$$
\begin{aligned}
1_{\mathcal{A}} & =\sum_{j=1}^{n} a_{j}\left(\left[1_{\mathcal{A}} \otimes \mu_{j}\right]\left(a \otimes 1_{\mathbb{C}}\right)-\lambda \mu_{j} 1_{\mathcal{A}}\right) \\
& =\sum_{j=1}^{n} a_{j}\left(\mu_{j} a-\mu_{j} \lambda 1_{\mathcal{A}}\right)=\sum_{j=1}^{n} a_{j} \mu_{j}\left(a-\lambda 1_{\mathcal{A}}\right) \\
& =b\left(a-\lambda 1_{\mathcal{A}}\right)
\end{aligned}
$$

and this is equivalent to $\lambda \notin \sigma_{\mathcal{A}}^{\text {left }}(a)$. Then $\sigma^{\text {left }}\left(a \otimes 1_{\mathbb{C}}\right)=\sigma_{\mathcal{A}}^{\text {left }}(a)$. If $\pi_{i}(a)$ $=a_{i}$ then Proposition 2.6 implies

$$
\sigma_{\mathcal{A}}^{\text {left }}(a)=\bigcup_{i \in I} \sigma_{\mathcal{A}_{i}}^{\text {left }}\left(a_{i}\right)
$$

Since $a \in \mathcal{A}$ is left invertible if and only if $0 \notin \sigma^{\text {left }}(a)=\bigcup_{i \in I} \sigma_{\mathcal{A}_{i}}^{\text {left }}\left(a_{i}\right)$, this is equivalent to $0 \notin \sigma_{\mathcal{A}_{i}}^{\text {left }}\left(a_{i}\right)$ for all $i$. Hence, $a \in \mathcal{A}$ is left invertible if and only if every $a_{i}$ is left invertible in $\mathcal{A}_{i}$.

3. Algebra-valued spectra. In this section we use the results in $\S 2$ with $E$ an lmc algebra to obtain invertibility results. We consider a $\in \mathcal{A} \widehat{\otimes}_{\tau} \mathcal{B}$ where $\tau$ is a uniform tensor norm and we suppose that $\mathcal{A} \widehat{\otimes}_{\tau} \mathcal{B}$ itself is an lmc algebra.

To obtain our results we need to extend a number of Banach algebra results in [9] to Q-algebras. The extensions are fairly straightforward, except for the following lemma, which is used to extend [9, Proposition 11]. We include the other results without proof. In the following lemma $\partial A$ denotes the boundary of the set $A$.

Lemma 3.1. Let $\mathcal{A}$ be a $Q$-algebra and let $z \in \partial \mathcal{A}_{\text {inv }}$. Then there exists a continuous multiplicative seminorm $p$ on $\mathcal{A}$ and a net $\left(z_{\alpha}\right)_{\alpha} \subseteq \mathcal{A}$ such that $p\left(z_{\alpha}\right)=1$ for all $\alpha$ and

$$
\lim _{\alpha} p\left(z_{\alpha} z\right)=0=\lim _{\alpha} p\left(z z_{\alpha}\right) .
$$

Proof. Since $z \in \partial \mathcal{A}_{\text {inv }}$, there exists a net $\left(r_{\alpha}\right)_{\alpha} \subseteq \mathcal{A}_{\text {inv }}$ such that $\lim _{\alpha} r_{\alpha}=z$. Since $\mathcal{A}$ is a Q-algebra there exist $\varepsilon>0$ and a continuous multiplicative seminorm $p$ such that $\left\{a \in \mathcal{A}: p\left(a-1_{\mathcal{A}}\right)<\varepsilon\right\} \subseteq \mathcal{A}_{\text {inv }}$. Suppose $\left(p\left(r_{\alpha}^{-1}\right)\right)_{\alpha}$ is bounded. Then

$$
p\left(r_{\alpha}^{-1}\left(z-r_{\alpha}\right)\right) \leq p\left(r_{\alpha}^{-1}\right) p\left(z-r_{\alpha}\right) \rightarrow 0 .
$$

Since $r_{\alpha}^{-1}\left(z-r_{\alpha}\right)=r_{\alpha}^{-1} z-1_{\mathcal{A}}$ we have $r_{\alpha}^{-1} z \in \mathcal{A}_{\text {inv }}$ for $\alpha$ sufficiently large. Hence $z=r_{\alpha}\left(r_{\alpha}^{-1} z\right) \in \mathcal{A}_{\text {inv }}$. Since $\mathcal{A}$ is a Q-algebra, $\mathcal{A}_{\text {inv }}$ is open 
and $\mathcal{A}_{\text {inv }} \cap \partial \mathcal{A}_{\text {inv }}=\emptyset$. This is a contradiction and, by taking a subnet if necessary, we may suppose $\lim _{\alpha} p\left(r_{\alpha}^{-1}\right)=\infty$.

If $z_{\alpha}:=r_{\alpha}^{-1} / p\left(r_{\alpha}^{-1}\right)$ then $p\left(z_{\alpha}\right)=1$ for all $\alpha$. Moreover

$$
z z_{\alpha}=\frac{z r_{\alpha}^{-1}}{p\left(r_{\alpha}^{-1}\right)}=\frac{1_{\mathcal{A}}+z r_{\alpha}^{-1}-r_{\alpha} r_{\alpha}^{-1}}{p\left(r_{\alpha}^{-1}\right)}=\frac{1_{\mathcal{A}}}{p\left(r_{\alpha}^{-1}\right)}+\left(z-r_{\alpha}\right) z_{\alpha}
$$

for all $\alpha$. Hence

$$
p\left(z z_{\alpha}\right) \leq \frac{1}{p\left(r_{\alpha}^{-1}\right)}+p\left(z-r_{\alpha}\right) p\left(z_{\alpha}\right) \rightarrow 0
$$

as $\alpha \rightarrow \infty$. In the same way $p\left(z_{\alpha} z\right) \rightarrow 0$ as $\alpha \rightarrow \infty$.

We now state the remaining results we require (the proof of (1) requires Lemma 3.1). The references are to the proofs for the Banach algebra cases.

[9, Proposition 11] Let $\left(a_{i}\right)_{i \in I}$ and $\left(b_{j}\right)_{j \in J}$ denote subsets of the Qalgebra $\mathcal{A}$. If $a_{i} a_{k}=a_{k} a_{i}$ and $a_{i} b_{j}=b_{j} a_{i}$ for all $i, k \in I$ and $j \in J$ then

$$
\pi_{J}\left(\sigma_{\mathrm{H}}^{\text {left }}\left(\left(a_{i}\right)_{i \in I},\left(b_{j}\right)_{j \in J}\right)\right)=\sigma_{\mathrm{H}}^{\text {left }}\left(\left(b_{j}\right)_{j \in J}\right)
$$

( $\pi_{J}$ denotes the usual projection).

[9, Proposition 13, Lemma 14] Let $\mathcal{A}$ be a unital commutative lmc algebra, $\mathcal{B}$ a unital lmc algebra and $\tau$ a uniform tensor norm such that $\mathcal{A} \widehat{\otimes}_{\tau} \mathcal{B}$ is an lmc algebra. If $a \in \mathcal{A} \widehat{\otimes}_{\tau} \mathcal{B}, \mu \in \mathbb{C}$ and $h \in \mathfrak{M}(\mathcal{A})$ then the ideals generated by

$$
\left\{\left[\left(a-h(a) 1_{\mathcal{A}}\right) \otimes 1_{\mathcal{B}}\right]_{a \in \mathcal{A}}, \mathrm{a}-\mu\left(1_{\mathcal{A}} \otimes 1_{\mathcal{B}}\right)\right\}
$$

and

$$
\left\{\left[\left(a-h(a) 1_{\mathcal{A}}\right) \otimes 1_{\mathcal{B}}\right]_{a \in \mathcal{A}}, 1_{\mathcal{A}} \otimes\left(\left[h \otimes I_{\mathcal{B}}\right](\mathrm{a})-\mu 1_{\mathcal{B}}\right)\right\}
$$

coincide.

$$
\text { If }\left(a_{i}\right)_{i \in I} \subseteq \mathcal{A},\left(b_{j}\right)_{j \in J} \subseteq \mathcal{B} \text { then }
$$

$$
\sigma_{\mathrm{H}}^{\text {left }}\left(\left(a_{i} \otimes 1_{\mathcal{B}}\right)_{i \in I},\left(1_{\mathcal{A}} \otimes b_{j}\right)_{j \in J}\right)=\sigma_{\mathrm{H}}^{\text {left }}\left(\left(a_{i}\right)_{i \in I}\right) \times \sigma_{\mathrm{H}}^{\text {left }}\left(\left(b_{j}\right)_{j \in J}\right) .
$$

Proposition 3.2. Let $\mathcal{A}$ be a commutative unital lmc algebra, $\mathcal{B}$ a unital lmc algebra and $\tau$ a uniform tensor norm such that $\mathcal{A} \widehat{\otimes}_{\tau} \mathcal{B}$ is a $Q$ algebra. If $\mathrm{a} \in \mathcal{A} \widehat{\otimes}_{\tau} \mathcal{B}$ then

$$
\sigma_{\mathcal{A} \tilde{\otimes}_{\tau} \mathcal{B}}^{\text {left }}(\mathrm{a})=\bigcup_{h \in \mathfrak{M}(\mathcal{A})} \sigma_{\mathcal{B}}^{\text {left }}\left(\left[h \otimes I_{\mathcal{B}}\right](\mathrm{a})\right)
$$

Proof. Since $h \otimes I_{\mathcal{B}}$ is a non-zero algebra homomorphism for all $h \in \mathfrak{M}(\mathcal{A})$ we have $\left[h \otimes I_{\mathcal{B}}\right]\left(1_{\mathcal{A}} \otimes 1_{\mathcal{B}}\right)=1_{\mathcal{B}}$ and

Hence

$$
\sigma_{\mathcal{B}}^{\text {left }}\left(\left[h \otimes I_{\mathcal{B}}\right](\mathrm{a})\right) \subseteq \sigma_{\mathcal{A} \widehat{\otimes}_{\tau} \mathcal{B}}^{\text {left }}(\mathrm{a}) .
$$

$$
\bigcup_{h \in \mathfrak{M}(\mathcal{A})} \sigma_{\mathcal{B}}^{\text {left }}\left(\left[h \otimes I_{\mathcal{B}}\right](\mathrm{a})\right) \subseteq \sigma_{\mathcal{A} \widehat{\otimes}_{\tau} \mathcal{B}}^{\text {left }}(\mathrm{a})
$$


Now suppose $\mu \in \sigma_{\mathcal{A} \widehat{\otimes}_{\tau} \mathcal{B}}^{\text {left }}\left(\right.$ a). Since the mapping $\mathcal{A} \rightarrow \mathcal{A} \widehat{\otimes}_{\tau} \mathcal{B}$ given by $a \mapsto a \otimes 1_{\mathcal{B}}$ is a non-zero continuous algebra homomorphism Lemma 2.2 implies that each element of $\sigma_{\mathrm{H}}^{\text {left }}\left(\left(a \otimes 1_{\mathcal{B}}\right)_{a \in \mathcal{A}}\right)$ belongs to $\mathfrak{M}(\mathcal{A})$. Since $\mathcal{A}$ is commutative, the system $\left\{\left(a \otimes 1_{\mathcal{B}}\right)_{a \in \mathcal{A}}, \mathrm{a}\right\} \subseteq \mathcal{A} \widehat{\otimes}_{\tau} \mathcal{B}$ satisfies the commutativity relations in (1). Hence by (1) there exists $h \in \mathfrak{M}(\mathcal{A})$ such that

$$
\left((h(a))_{a \in \mathcal{A}}, \mu\right) \in \sigma_{\mathrm{H}}^{\text {left }}\left(\left(a \otimes 1_{\mathcal{B}}\right)_{a \in \mathcal{A}}, \mathrm{a}\right) .
$$

By (2) and (3),

$$
\begin{aligned}
\left((h(a))_{a \in \mathcal{A}}, \mu\right) & \in \sigma_{\mathrm{H}}^{\text {left }}\left(\left(a \otimes 1_{\mathcal{B}}\right)_{a \in \mathcal{A}}, 1_{\mathcal{A}} \otimes\left(\left[h \otimes I_{\mathcal{B}}\right](\mathrm{a})\right)\right) \\
& =\sigma_{\mathrm{H}}^{\text {left }}\left((a)_{a \in \mathcal{A}}\right) \times \sigma_{\mathcal{B}}^{\text {left }}\left(\left[h \otimes I_{\mathcal{B}}\right](\mathrm{a})\right) .
\end{aligned}
$$

Hence $\mu \in \sigma_{\mathcal{B}}^{\text {left }}\left(\left[h \otimes I_{\mathcal{B}}\right](\mathrm{a})\right)$.

Proposition 3.2 generalizes part of [9, Proposition 20] from unital Banach algebras to Q-algebras, and the remaining part is generalized in Proposition 4.5 below. Clearly Proposition 3.2 also holds for right spectra. Combining the results for right and left spectra we obtain an analogous result for the usual spectrum and recover a special case of results due to M. Fragoulopoulou [13, Proposition 6.9] and [14, Proposition 4.13].

REMARK 3.3. If $\mathcal{A} \widehat{\otimes}_{\tau} \mathcal{B}$ is a Q-algebra then, since $\tau$ is a uniform tensor norm, there exists a continuous multiplicative seminorm $p$ on $\mathcal{A}$ such that $a \otimes 1_{\mathcal{B}} \in\left(\mathcal{A} \widehat{\otimes}_{\tau} \mathcal{B}\right)_{\text {inv }}$ for all $a$ such that $p\left(a-1_{\mathcal{A}}\right)<1$. If $\mathrm{b} \in \mathcal{A} \widehat{\otimes}_{\tau} \mathcal{B}$ satisfies

$$
\left(a \otimes 1_{\mathcal{B}}\right) \mathrm{b}=\mathrm{b}\left(a \otimes 1_{\mathcal{B}}\right)=1_{\mathcal{A}} \otimes 1_{\mathcal{B}}
$$

and $\varphi \in \mathcal{B}^{\prime}$ is chosen so that $\varphi\left(1_{\mathcal{B}}\right)=1$ then, by density and continuity,

$$
\left(1_{\mathcal{A}} \otimes \varphi\right)\left(\left(a \otimes 1_{\mathcal{B}}\right) \mathrm{b}\right)=a\left(1_{\mathcal{A}} \otimes \varphi\right)(\mathrm{b})=\left(1_{\mathcal{A}} \otimes \varphi\right)(\mathrm{b}) a=1_{\mathcal{A}}
$$

and $\left\{a \in \mathcal{A}: p\left(a-1_{\mathcal{A}}\right)<1\right\} \subseteq \mathcal{A}_{\text {inv }}$. On interchanging the roles of $\mathcal{A}$ and $\mathcal{B}$ we thus see that if $\mathcal{A} \widehat{\otimes}_{\tau} \mathcal{B}$ is a Q-algebra then so also are $\mathcal{A}$ and $\mathcal{B}$. Our next result shows that the converse is true if and only if pointwise invertibility implies invertibility.

TheOREM 3.4. If $\mathcal{A}$ is a commutative $Q$-algebra, $\mathcal{B}$ is a $Q$-algebra and $\tau$ is a uniform tensor norm such that $\mathcal{A} \widehat{\otimes}_{\tau} \mathcal{B}$ is an lmc algebra then the following are equivalent:

(1) $\mathcal{A} \widehat{\otimes}_{\tau} \mathcal{B}$ is a Q-algebra.

(2) If $\mathrm{a} \in \mathcal{A} \widehat{\otimes}_{\tau} \mathcal{B}$ then

$$
\sigma_{\mathcal{A} \tilde{\otimes}_{\tau} \mathcal{B}}^{\text {left }}(\mathrm{a})=\bigcup_{h \in \mathfrak{M}(\mathcal{A})} \sigma_{\mathcal{B}}^{\text {left }}\left(\left[h \otimes I_{\mathcal{B}}\right](\mathrm{a})\right)
$$

Proof. We have $(1) \Rightarrow(2)$ by Proposition 3.2. Suppose (2) holds. Since $\mathcal{A}$ is a Q-algebra, $\mathfrak{M}(\mathcal{A})$ is an equicontinuous subset of $\mathcal{A}^{\prime}[20$, p. 187]. Hence there exists a continuous multiplicative seminorm $p$ on $\mathcal{A}$ such that 
$|h(a)| \leq p(a)$ for all $h \in \mathfrak{M}(\mathcal{A})$ and all $a \in \mathcal{A}$. Since $\mathcal{B}$ is a Q-algebra there exists a continuous multiplicative seminorm $q$ on $\mathcal{B}$ such that $\left\{1_{\mathcal{B}}+y\right.$ : $q(y)<1\} \subseteq \mathcal{B}_{\text {inv }}$.

Now suppose $\mathrm{a} \in \mathcal{A} \widehat{\otimes}_{\tau} \mathcal{B}$ and $p \otimes_{\tau} q(\mathrm{a})<1$. If $h \in \mathfrak{M}(\mathcal{A})$ then

$$
\left[h \otimes I_{\mathcal{B}}\right]\left(1_{\mathcal{A}} \otimes 1_{\mathcal{B}}+\mathrm{a}\right)=1_{\mathcal{B}}+\left[h \otimes I_{\mathcal{B}}\right](\mathrm{a}) .
$$

Next,

$$
q\left(\left[h \otimes I_{\mathcal{B}}\right](\mathrm{a})\right)=\sup _{\substack{\varphi \in \mathcal{B}^{\prime} \\ \varphi \in B_{q}^{\circ}}}\left|\varphi\left(\left[h \otimes I_{\mathcal{B}}\right](\mathrm{a})\right)\right|=\sup _{\substack{\varphi \in \mathcal{B}^{\prime} \\ \varphi \in B_{q}^{\circ}}}|[h \otimes \varphi](\mathrm{a})| \leq p \otimes_{\tau} q(\mathrm{a})<1 .
$$

Hence $1_{\mathcal{B}}+\left[h \otimes I_{\mathcal{B}}\right](\mathrm{a}) \in \mathcal{B}_{\text {inv }}$ and, by $(2)$, a $\in\left(\mathcal{A} \widehat{\otimes}_{\tau} \mathcal{B}\right)_{\text {inv }}$. Hence $\mathcal{A} \widehat{\otimes}_{\tau} \mathcal{B}$ is a Q-algebra.

The following result for the projective tensor product is due to $\mathrm{H}$. A. Smith [25, Theorem 3] and to A. Mallios [19, Proposition 4.2] and [20, Lemma, p. 412] for more general tensor norms when $\mathcal{A}$ and $\mathcal{B}$ are both commutative. Special cases when $\mathcal{A}=\mathcal{C}(K), K$ compact Hausdorff, and $\mathcal{A}=$ $\mathcal{C}^{\infty}(X), X$ a finite-dimensional compact manifold, and $\mathcal{B}$ is non-commutative are considered in [19, Lemma 2.1] and [15, Example 3.4].

Theorem 3.5. If $\mathcal{A}$ is a commutative Fréchet $Q$-algebra, $\mathcal{B}$ is a Fréchet $Q$-algebra, and $\tau$ is a uniform tensor norm that satisfies the projective limit condition and such that $\mathcal{A} \widehat{\otimes}_{\tau} \mathcal{B}$ is a representable lmc algebra, then $\mathcal{A} \widehat{\otimes}_{\tau} \mathcal{B}$ is a Q-algebra.

Proof. By Proposition 1.3, condition (2) of Theorem 3.4 is satisfied. Hence $\mathcal{A} \widehat{\otimes}_{\tau} \mathcal{B}$ is a Q-algebra.

Examples can now be obtained by looking at our remarks after Definition 1.2. For instance $\mathcal{A} \widehat{\otimes}_{\pi} \mathcal{B}$ is a Q-algebra whenever $\mathcal{A}$ is a commutative Fréchet Q-algebra and $\mathcal{B}$ is an arbitrary Fréchet Q-algebra.

\section{The Gelfand transform}

Definition 4.1. Let $\mathcal{A}$ be an lmc algebra, $E$ a complete locally convex space and $\tau$ a uniform tensor norm. For each a $\in \mathcal{A} \widehat{\otimes}_{\tau} E$ we define its Gelfand transform:

$$
\widehat{\mathrm{a}}: \mathfrak{M}(\mathcal{A}) \rightarrow E, \quad \widehat{\mathrm{a}}(h)=\left[h \otimes I_{E}\right](\mathrm{a}) .
$$

We endow $\mathfrak{M}(\mathcal{A})$ with the topology induced by $\sigma\left(\mathcal{A}^{\prime}, \mathcal{A}\right)$.

Proposition 4.2. Let $\mathcal{A}$ be a Q-algebra, $E$ a complete locally convex space and $\tau$ a uniform tensor norm. If $\mathrm{a} \in \mathcal{A} \widehat{\otimes}_{\tau} E$, then $\widehat{\mathrm{a}} \in \mathcal{C}(\mathfrak{M}(\mathcal{A}), E)$.

Proof. Suppose $\left(h_{\alpha}\right)_{\alpha} \subseteq \mathfrak{M}(\mathcal{A})$ and $h_{\alpha} \rightarrow h$ as $\alpha \rightarrow \infty$. Let $q$ be any continuous seminorm on $E$ and $\varepsilon>0$ be arbitrary. Since $\mathcal{A}$ is a Q-algebra, 
$\mathfrak{M}(\mathcal{A}) \otimes\left\{I_{E}\right\}$ is an equicontinuous subset of $\mathcal{L}\left(\mathcal{A} \widehat{\otimes}_{\tau} E ; E\right)$. Hence we can find a continuous seminorm $p$ on $\mathcal{A} \widehat{\otimes}_{\tau} E$ such that

$$
q\left(\left[g \otimes I_{E}\right](\mathrm{b})\right) \leq p(\mathrm{~b})
$$

for all $\mathrm{b} \in \mathcal{A} \widehat{\otimes}_{\tau} E$ and all $g \in \mathfrak{M}(\mathcal{A})$. If $\mathrm{a}:=\sum_{i=1}^{n} a_{i} \otimes x_{i} \in \mathcal{A} \otimes E$ then $\widehat{\mathrm{a}}(h)=\sum_{i=1}^{n} h\left(a_{i}\right) x_{i}$ is clearly continuous. If a is arbitrary we can choose $\mathrm{b} \in \mathcal{A} \otimes E$ such that $p(\mathrm{a}-\mathrm{b})<\varepsilon$. Now choose $\alpha_{0}$ such that

$$
q\left(\left[h_{\alpha} \otimes I_{E}\right](\mathrm{b})-\left[h \otimes I_{E}\right](\mathrm{b})\right)<\varepsilon
$$

for all $\alpha \geq \alpha_{0}$. Then

$$
\begin{aligned}
q\left(\left[h_{\alpha} \otimes I_{E}\right](\mathrm{a})-\left[h \otimes I_{E}\right](\mathrm{a})\right) \leq & q\left(\left[h_{\alpha} \otimes I_{E}\right](\mathrm{b})-\left[h \otimes I_{E}\right](\mathrm{b})\right) \\
& +q\left(\left[\left(h_{\alpha}-h\right) \otimes I_{E}\right](\mathrm{b}-\mathrm{a})\right) \\
\leq & \varepsilon+2 p(\mathrm{a}-\mathrm{b}) \leq 3 \varepsilon .
\end{aligned}
$$

Hence $\widehat{a} \in \mathcal{C}(\mathfrak{M}(\mathcal{A}), E)$.

Definition 4.3. If $\mathcal{A}$ is a $\mathrm{Q}$-algebra, $E$ is a complete locally convex space and $\tau$ is a uniform tensor norm, the Gelfand mapping ${ }^{\wedge}: \mathcal{A} \widehat{\otimes}_{\tau} E \rightarrow$ $\mathcal{C}(\mathfrak{M}(\mathcal{A}), E)$ is defined by $\widehat{a}(h)=\left[h \otimes I_{E}\right](\mathrm{a})$.

Proposition 4.4. If $\mathcal{A}$ is a Q-algebra, $E$ is a complete locally convex space and $\tau$ is a uniform tensor norm then the Gelfand mapping is a continuous linear mapping from $\mathcal{A} \widehat{\otimes}_{\tau} E$ into $\mathcal{C}(\mathfrak{M}(\mathcal{A}), E)$. If $\mathcal{B}$ is a complete lmc algebra and $\mathcal{A} \widehat{\otimes}_{\tau} \mathcal{B}$ is an lmc algebra, then the Gelfand mapping is an algebra homomorphism.

Proof. The Gelfand mapping is easily seen that to be linear. The estimate (5) in the previous proposition shows that it is continuous. When $\mathcal{B}$ is an algebra and $\mathcal{A} \widehat{\otimes}_{\tau} \mathcal{B}$ is an lmc algebra then the Gelfand mapping is easily seen to be a homomorphism from $\mathcal{A} \otimes E$ into $\mathcal{C}(\mathfrak{M}(\mathcal{A}), E)$. Continuity and density can be applied to complete the proof.

Proposition 4.5. If $\mathcal{A}$ is a commutative unital lmc algebra, $\mathcal{B}$ is a unital lmc algebra and $\tau$ is a uniform tensor norm such that $\mathcal{A} \widehat{\otimes}_{\tau} \mathcal{B}$ is a $Q$-algebra then for $\mathrm{a} \in \mathcal{A} \widehat{\otimes}_{\tau} \mathcal{B}$ the following are equivalent:

(1) a is left invertible in $\mathcal{A} \widehat{\otimes}_{\tau} \mathcal{B}$.

(2) $\widehat{a}(h)$ is left invertible in $\mathcal{B}$ for every $h \in \mathfrak{M}(\mathcal{A})$.

(3) $\widehat{a}$ is left invertible in $\mathcal{C}(\mathfrak{M}(\mathcal{A}), \mathcal{B})$.

Proof. By Proposition 3.2, (1) $\Leftrightarrow(2)$. By Proposition 4.4, a $\mapsto \widehat{a}$ is an algebra homomorphism and $1_{\mathcal{A}} \widehat{\otimes} 1_{\mathcal{B}}(h)=I_{\mathcal{B}}$ for all $h \in \mathfrak{M}(\mathcal{A})$. Hence the Gelfand transform maps left invertible elements to left invertible elements and $(1) \Rightarrow(3)$. Since we always have $(3) \Rightarrow(2)$ this completes the proof.

EXAMPLE 4.6. Let $E$ be Fréchet-Schwartz space whose topology is generated by an increasing sequence $\left(p_{n}\right)_{n \in \mathbb{N}}$ of seminorms such that each $\widehat{E}_{n}$, 
which is a Banach space, has the approximation property. Take $K \subseteq E$ compact, balanced and polynomially convex and $\mathcal{B}$ a unital Banach algebra. Let $\mathcal{H}(K, \mathcal{B})$ denote the space of $\mathcal{B}$-valued holomorphic germs on $K$. We have the following representation (see [4]):

$$
\left(\mathcal{H}(K, \mathcal{B}), \tau_{\omega}\right) \cong\left(\mathcal{H}(K), \tau_{\omega}\right) \widehat{\otimes}_{\varepsilon} \mathcal{B} .
$$

Moreover, since $K$ is polynomially convex, $\mathfrak{M}(\mathcal{H}(K)) \cong K$ by means of the identification $h(f)=f(k)$. Both $\mathcal{H}(K)$ and $\mathcal{H}(K, \mathcal{B})$ are Q-algebras, since they are inductive limits of Banach algebras.

As in Example 1.4, $\left[h_{k} \otimes I_{\mathcal{B}}\right](\mathrm{a})=\mathrm{a}(k)$ for all $\mathrm{a} \in \mathcal{H}(K) \widehat{\otimes}_{\varepsilon} \mathcal{B}$ and $k \in K$. By Proposition 3.2, $F \in \mathcal{H}(K, \mathcal{B})$ is left invertible if and only if $F(k)$ is left invertible in $\mathcal{B}$ for all $k \in K$.

Since the Gelfand mapping $\widehat{\wedge} \mathcal{H}(K, \mathcal{B}) \rightarrow \mathcal{C}(K, \mathcal{B})$ is the inclusion mapping, Proposition 4.5 implies that $F$ is left invertible in $\mathcal{H}(K, \mathcal{B})$ if and only if it is left invertible in $\mathcal{C}(K, \mathcal{B})$.

\section{References}

[1] G. R. Allan, On one-sided inverses in Banach algebras of holomorphic vector-valued functions, J. London Math. Soc. 42 (1967), 463-470.

[2] - Holomorphic vector-valued functions on a domain of holomorphy, ibid., 509-513.

[3] R. Arens, Dense inverse limit rings, Michigan Math. J. 5 (1958), 169-182.

[4] K. D. Bierstedt, J. Bonet and A. Peris, Vector-valued holomorphic germs on FréchetSchwartz spaces, Proc. Roy. Irish Acad. Sect. A 94 (1994), 31-46.

[5] M. Chidami et R. El Harti, Calcul fonctionnel holomorphe en dimension infinie dans les lmca, Rend. Circ. Mat. Palermo (2) 48 (1999), 541-548.

[6] I. G. Craw, Envelopes of holomorphy for holomorphic vector-valued functions, J. London Math. Soc. (2) 4 (1971), 327-330.

[7] A. Defant and K. Floret, Tensor Norms and Operator Ideals, North-Holland Math. Stud. 176, North-Holland, Amsterdam, 1993.

[8] S. Dierolf and K. Aye Aye, On the spectra of elements in certain algebras of vectorvalued functions and sequences, Extracta Math. 13 (1998), 35-40.

[9] S. Dineen, R. E. Harte and C. Taylor, Spectra of tensor product elements I: Basic theory, Proc. Roy. Irish Acad. Sect. A 101 (2001), 177-196.

[10] - - - - , Spectra of tensor product elements II: Polynomial extensions, ibid., 197200.

[11] —, - - - Spectra of tensor product elements III: Holomorphic properties, ibid., to appear.

[12] S. Dineen and J. Mujica, The Approximation Property for spaces of holomorphic functions on infinite dimensional spaces, preprint.

[13] M. Fragoulopoulou, Symmetric topological ${ }^{*}$-algebras. Applications, Schriftenreihe Math. Univ. Münster 3. Ser. 9 (1993), 1-124.

[14] —, Tensor products of enveloping locally $C^{*}$-algebras, ibid. 21 (1997), 1-81.

[15] M. Fragoulopoulou, On hermitian algebras, in: Proc. of ICTAA 2 (Rabat, 2000), to appear.

[16] H. Goldmann, Uniform Fréchet Algebras, North-Holland Math. Stud. 162, NorthHolland, Amsterdam, 1990. 
[17] J. Horváth, Topological Vector Spaces and Distributions, I, Addison-Wesley, 1966.

[18] H. Jarchow, Locally Convex Spaces, Teubner, Stuttgart, 1981.

[19] A. Mallios, On the spectrum of a topological tensor product of locally convex algebras, Math. Ann. 154 (1964), 171-180.

[20] —, Topological Algebras. Selected Topics, North-Holland, Amsterdam, 1986.

[21] —, Continuous vector bundles over topological algebras, J. Math. Anal. Appl. 113 (1986), 245-254.

[22] A. E. Michael, Locally multiplicatively-convex topological algebras, Mem. Amer. Math. Soc. 11 (1952).

[23] J. Mujica, Domains of holomorphy in (DFC)-spaces, in: Functional Analysis, Holomorphy, and Approximation Theory, S. Machado (ed.), Lecture Notes in Math. 843, Springer, 1981, 500-533.

[24] M. Schottenloher, A Cartan-Thullen theorem for domains spread over DFM-spaces, J. Reine Angew. Math. 345 (1983), 201-220.

[25] H. A. Smith, Tensor products of locally convex algebras, Proc. Amer. Math. Soc. 17 (1966), 124-132.

[26] L. Waelbroeck, Topological Vector Spaces and Algebras, Lecture Notes in Math. 230, Springer, Berlin, 1973.

[27] S. Warner, Polynomial completeness in locally multliplicatively-convex algebras, Duke Math. J. 23 (1956), 1-11.

Department of Mathematics

University College Dublin

Belfield

Dublin 4, Ireland

E-mail: Sean.Dineen@ucd.ie
Departamento de Análisis Matemático Universidad de Valencia

C/ Doctor Moliner 50 46100 Burjasot (Valencia), Spain E-mail: Pablo.Sevilla@uv.es

Received September 17, 2001

Revised version June 13, 2002 